\title{
Alterações no sistema auditivo de crianças e adolescentes com síndrome da imunodeficiência adquirida: revisão sistemática
}

\author{
Changes in the auditory system of children and adolescents with acquired immunodeficiency \\ syndrome: systematic review \\ Cambios en el sistema auditivo de niños y adolescentes con síndrome de inmunodeficiencia \\ adquirida: revisión
}

Recebido: 14/12/2021 | Revisado: 21/12/2021 | Aceito: 02/01/2021 | Publicado: 02/01/2022

\author{
Ariele Júllian Santos Lima \\ ORCID: https://orcid.org/0000-0003-0300-7999 \\ Universidade Ceuma, Brasil \\ E-mail:arielyjullian@ hotmail.com \\ Thamyres Souza Nascimento \\ ORCID:https://orcid.org/0000-0002-1038-9609 \\ Universidade Ceuma, Brasil \\ E-mail:thamyres.tsn25@gmail.com \\ Érica Alessandra Caldas \\ ORCID: https://orcid.org/0000-0002-6507-4735 \\ Universidade Federal do Maranhão, Brasil \\ E-mail:ericaacaldas@hotmail.com
}

\begin{abstract}
Resumo
Objetivo: Realizar uma revisão sistemática de literatura sobre as principais alterações no sistema auditivode crianças e adolescentes com AIDS. Metodologia: Trata-se de um estudo de revisão sistemática da literatura, que tem aseguinte pergunta norteadora: quais são as alterações no sistema auditivo encontradas em crianças e adolescentes acometidos pela Síndrome da Imunodeficiência Adquirida? A estratégia de busca da pesquisa se deu através da combinação de cinco descritores extraídos do DeCS (Descritores em Ciências da Saúde) em dois idiomas: português e inglês. Foram utilizados os seguintes descritores: "perda auditiva", "síndrome da imunodeficiência adquirida", "crianças", "adolescentes" e "perda auditiva central". Os critérios de inclusão foram: artigos originais, publicações a partir de 2011, nos idiomas inglês e português que envolviam a Síndrome da Imunodeficiência Adquirida no público infantil e adolescente. Resultados: Foram selecionados 10 artigos que atenderam aos critérios de inclusão. Os estudos relataram alterações audiológicas a nível periférico e central. Em relação ao sistema auditivo periférico, a complicação mais descrita foi a perda auditiva e, apesar do público em questão ser susceptível aos diversos tipos desta patologia, a mais encontrada na literatura foi a perda auditiva condutiva. No que diz respeito ao nível central da audição, alterações no processamento auditivo central foram descritos nos estudos, pois o sistema nervoso central pode servir de reservatório para o HIV, afetando as vias auditivas centrais. Conclusão: As alterações auditivas em crianças e adolescentes acometidos pela Síndrome da Imunodeficiência Adquirida mais encontradas na literatura foram: perda auditiva do tipo condutiva e complicações no processamento auditivo central.
\end{abstract}

Palavras-chave:Perda auditiva; Síndrome da imunodeficiência adquirida; Crianças; Adolescentes; Perda auditiva central.

\begin{abstract}
Objective: To carry out a systematic literature review on the main alterations in the auditory system of children and adolescents with AIDS. Methodology: This is a systematic literature review study, which has the following guiding question: what are the alterations in the auditory system found in children and adolescents with Acquired Immune Deficiency Syndrome? The search search strategy was carried out by combining five descriptors extracted from DeCS (Descriptors in Health Sciences) in two languages: Portuguese and English. The following descriptors were used: "hearing loss", "acquired immunodeficiency syndrome", "children", "adolescents" and "central hearing loss". Inclusion criteria were: original articles, publications from 2011, in English and Portuguese that involved the Acquired Immunodeficiency Syndrome in children and adolescents. Results: 10 articles that met the inclusion criteria were selected. The studies reported audiological alterations at the peripheral and central levels. In relation to the peripheral auditory system, the most described complication was hearing loss and, although the public in question is susceptible to different types of this pathology, the most common one found in the literature was conductive hearing loss. With regard to the central level of hearing, alterations in central auditory processing were described in the studies, as the central nervous system can serve as a reservoir for HIV, affecting the central auditory pathways. Conclusion: The most common hearing alterations in children and adolescents affected by the Acquired
\end{abstract}


Immunodeficiency Syndrome in the literature were: conductive hearing loss and complications in central auditory processing.

Keywords:Hearing loss; Acquired immunodeficiency syndrome; Kids; Teenagers; Central hearing loss.

\section{Resumen}

Objetivo: Realizar una revisión bibliográfica sistemática sobre las principales alteraciones del sistema auditivo de niños y adolescentes con sida. Metodología: Se trata de un estudio de revisión sistemática de la literatura, que tiene la siguiente pregunta orientadora: ¿Cuáles son las alteraciones en el sistema auditivo encontradas en niños y adolescentes con Síndrome de Inmunodeficiencia Adquirida? La estrategia de búsqueda se llevó a cabo combinando cinco descriptores extraídos de DeCS (Descriptors in Health Sciences) en dos idiomas: portugués e inglés. Se utilizaron los siguientes descriptores: "hipoacusia", "síndrome de inmunodeficiencia adquirida", "niños", “adolescentes" y "hipoacusia central". Los criterios de inclusión fueron: artículos originales, publicaciones de 2011, en inglés y portugués, que involucraran el Síndrome de Inmunodeficiencia Adquirida en niños y adolescentes. Resultados: Se seleccionaron 10 artículos que cumplieron con los criterios de inclusión. Los estudios informaron alteraciones audiológicas a nivel periférico y central. En relación al sistema auditivo periférico, la complicación más descrita fue la hipoacusia y, aunque el público en cuestión es susceptible a diferentes tipos de esta patología, la más común encontrada en la literatura fue la hipoacusia conductiva. Con respecto al nivel central de la audición, en los estudios se describieron alteraciones en el procesamiento auditivo central, ya que el sistema nervioso central puede servir como reservorio del VIH, afectando las vías auditivas centrales. Conclusión: Las alteraciones auditivas más comunes en niños y adolescentes afectados por el Síndrome de Inmunodeficiencia Adquirida en la literatura fueron: hipoacusia conductiva y complicaciones en el procesamiento auditivo central.
\end{abstract}

Palabrasclave: Pérdida de la audición; Síndrome de inmunodeficiencia adquirida; Niños; Adolescentes; Hipoacusia central.

\title{
1. Introdução
}

A Síndrome da Imunodeficiência Adquirida (AIDS), causada pelo vírus da Imunodeficiência Humana (HIV) é considerada uma doença infectocontagiosa e sem cura que ataca o sistema imunológico, impedindo o organismo de combater infecções oportunistas (Santos et. al., 2019); (Matas et. al., 2017). Seu processo de infecção se dá através do contato com o sangue ou secreções de um indivíduo já infectado. Por conta dessa alteração no sistema imunológico, o portador do HIV está sujeito a diversos males como: hepatites virais, tuberculose, toxoplasmose, pneumonia e alguns tipos de câncer (Gregory et. al., 2013); (Buriti et. al., 2013); (Hortúa et. al., 2020).

Além dos males citados acima, a ação do vírus no sistema imunológico e o uso de medicamentos antirretrovirais podem causar alterações auditivas, sendo este último, o principal responsável por reduzir o avanço da doença, promovendo melhora no quadro clínico do paciente. No entanto, estudos apontam que o uso desses medicamentos aumentam as chances de perda auditiva em crianças por se tratarem de medicamentos ototóxicos. Esses são compostos por substâncias químicas que podem lesionar o sistema coclear e vestibular. (Oluwabusayo et. al., 2018); (Costa et. al., 2020); (Quidicomo et. al., 2013); (Smith et. al., 2017); (Minhas et. al., 2018); (Nakku et. al.,2017).

De acordo com Maro et. al. (2016), o HIV pode impactar diferentes estruturas do sistema auditivo de crianças, tanto periféricas (cóclea) quanto centrais. O sistema nervoso central pode servir de reservatório para o HIV, afetando as vias auditivas centrais, levando desta forma a alterações do Processamento Auditivo Central.

A perda auditiva destaca-se dentre as alterações que podem ser desenvolvidas em crianças contaminadas pelo HIV, porém podem ser encontradas ainda além das alterações no processamento auditivo central, otites, perfuração timpânicae zumbido. Alguns estudos avaliaram a ocorrência destas alterações em crianças portadoras do HIV, um deles refere à faixa de $64 \%$ para perda auditiva do tipo sensorioneural na amostra avaliada, afetando o sistema central e outro revela o índice predominante de $29 \%$ para perda do tipo condutiva em crianças, afetando o sistema periférico (Taipale et. al., 2011); (Ndoleriire et. al., 2013); (Peter et. al., 2020).

Autores destacam que crianças com HIV/AIDS são susceptíveis a alterações auditivas de diversos tipos (condutiva, sensorioneural e mista em diferentes faixas etárias durante a infância e em diversos períodos a partir do diagnóstico da 
AIDS.(Buriti et. al., 2014); (Romero et. al., 2017); (Sebothoma \&Shangase, 2018).

Levando em consideração a importância da integridade do sistema auditivo para o desenvolvimento da linguagem, aprendizagem e socialização, o diagnóstico precoce das alterações no sistema auditivo periférico e central favorece

o prognóstico minimizando as alterações no desenvolvimento infantil e melhorando a qualidade de vida. Desta forma, torna-se relevante a identificação e acompanhamento da saúde auditiva em crianças portadoras do HIV para que sejam promovidas estratégias que proporcionem o desempenho escolar, desenvolvendo habilidades de fala e linguagem que contribuam para o crescimento social das mesmas (Ndoleriire et. al., 2013); (Peter et. al., 2020).

Como contribuição ao entendimento sobre as alterações no sistema auditivo de crianças e adolescentes com síndrome da imunodeficiência adquirida o objetivo deste estudo foi investigar na literatura as principais alterações no sistema auditivo ocasionadas pela Síndrome da Imunodeficiência Adquirida no público infantil e adolescente.

\section{Metodologia}

A metodologia aplicada para a fundamentação deste trabalho foi a pesquisa de revisão sistemática. Este é um método descrito por De-La-Torre-Ugarte-Guanilo et. al. (2011) como "uma metodologia rigorosa proposta para: identificar os estudos sobre um tema em questão, aplicando métodos explícitos e sistematizados de busca".

Trata-se de um estudo que tem a seguinte pergunta norteadora: quais são as alterações no sistema auditivo encontradas em crianças acometidas pela Síndrome da Imunodeficiência Adquirida? Para critérios de inclusão neste trabalho utilizamos: artigos originais publicações a partir de 2011, nos idiomas inglês e português que envolviam a Síndrome da Imunodeficiência Adquirida no público infantil e adolescente. Foram excluídas da pesquisa publicações que se tratavam de revisões bibliográficas ou estudos de casos, artigos duplicados e artigos que não se encontravam disponíveis na integra.

A busca foi conduzida nos meses de maio a setembro de 2021, através de diferentes combinações entre cinco descritores extraídos do DeCS (Descritores em Ciências da Saúde): "perda auditiva”, "síndrome da imunodeficiência adquirida", "crianças", "adolescentes"e "perda auditiva central" nos idiomas português e inglês utilizando o operador booleano "e" para associação desses descritores.

As bases de dados científicas selecionadas foram: BVS (Biblioteca Virtual em Saúde), SciELO (ScientificElectronic Library Online), LILACS (Literatura Latino-Americana e do Caribe em Ciências da Saúde) e PubMed (US National Library of Medicine).

\section{ResultadoseDiscussão}

Na busca online foram identificados 890 estudos, após eliminação de estudos duplicados, resultaram em 504estudos. A seleção desses estudos foi conduzida em duas fases: leitura dos resumos eleitura de artigos completos, realizada por dois revisores de forma independente (AJSL e TSN), sendo utilizada uma terceira revisora (EAC) nos casos de discordância entre os dois primeiros revisores.

Um total de 312 estudos foram excluídos após a leitura de resumos, resultando em132 artigos para leitura na íntegra.Ao final da leitura dos estudos na íntegra, foram excluídos 122 artigos por não atenderem aos critérios de elegibilidade, resultando uma amostra final de 10 artigos (Figura 1). 
Figura 1.Fluxograma do processo de seleção dos estudos.

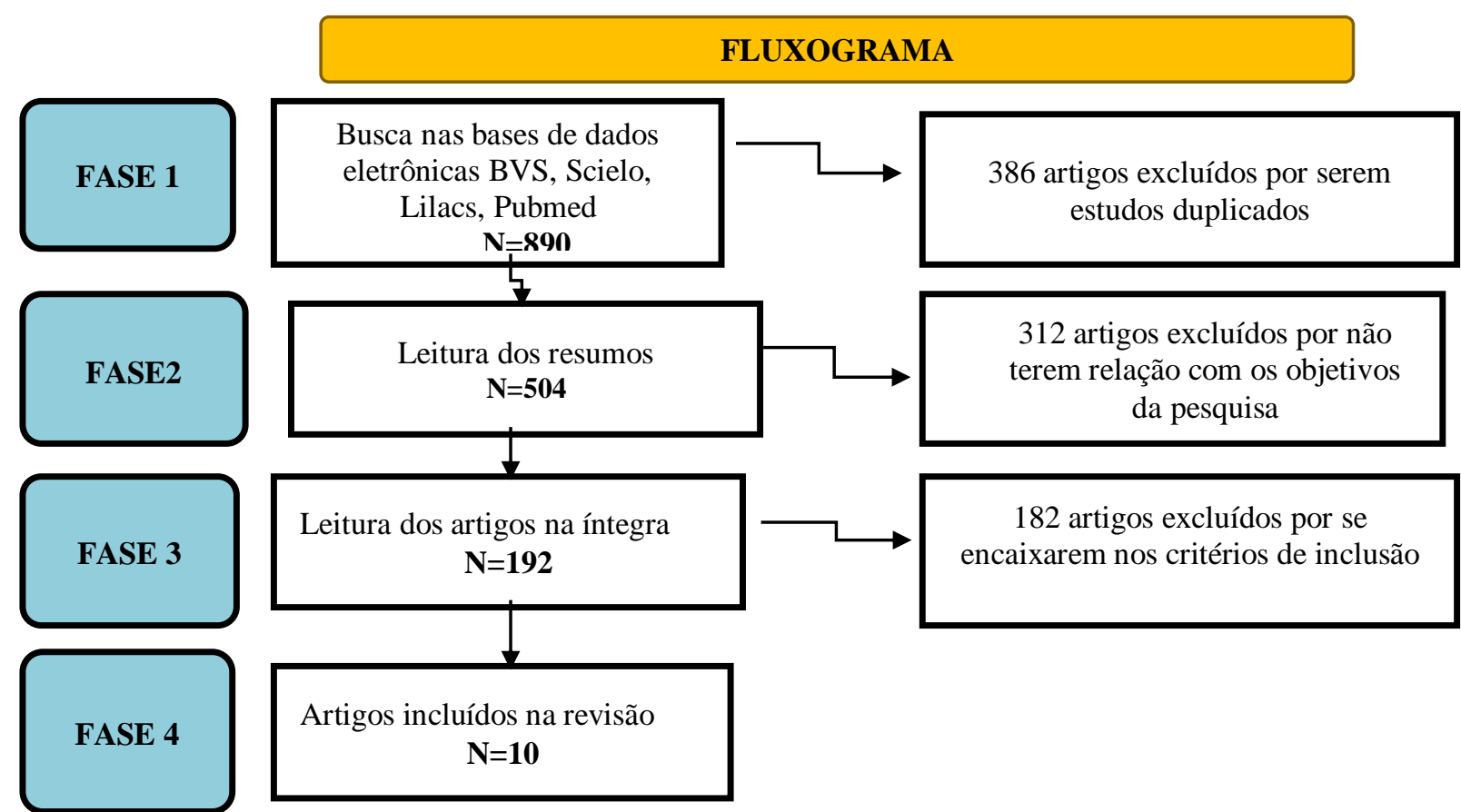

Fonte: Autores.

Os dados das características das publicações incluídas com relação a autor, ano da publicação, tipo de estudo, tamanho amostral, faixa etária da amostra e país de publicação estão demonstrados na Tabela 1.

Os artigos incluídos eram em sua maioria transversais (90\%), sendo apenas um deles (10\%) de caso controle. As datas de publicação apresentaram uma lacuna nos anos de 2018 e 2019, porém com interesse em publicação sobre o assunto praticamente constante durante os últimos 10 anos.O tamanho amostral dos estudos foi heterogêneo, variando entre 14 e 370 participantes. Dos estudos encontrados seis (60\%) são internacionais (Taipale et. al., 2011); (Ndoleriire et. al., 2013); (Peter et. al., 2020); (Nakku et. al., 2017); (Chao et. al., 2012); (Torre et. al., 2015), sendo que oito (80 \%) dos 10 incluíram crianças de até 12 anos (Buriti et. al., 2013); (Taipale et. al., 2011); (Ndoleriire et. al., 2013); (Peter et. al., 2020); (Buriti et. al., 2014); (Romero et. al., 2017); (Nakku et. al., 2017); (Chao et. al.,2012), os demais(20\%) incluíram crianças e adolescentes (Gregory et. al., 2013); (Torre et. al.,2015).

Tabela 1. Características das publicações incluídas.

\begin{tabular}{|c|c|c|c|c|}
\hline AUTOR/ ANO & TIPO DE ESTUDO & $\begin{array}{l}\text { AMOSTRAGEM } \\
(\mathrm{N})\end{array}$ & FAIXA ETÁRIA & $\begin{array}{c}\text { PAÍS } \\
\text { REALIZAÇÃO }\end{array}$ \\
\hline Taipale et. al., 2011 & Caso Controle & 78 & Até 11 anos & Angola \\
\hline Chao et. al.,2012 & Transversal & 139 & A partir de 4 anos & Áustria \\
\hline Ndoleriire C. et al., 2012 & Transversal & 370 & $6 \mathrm{~m}$ a 5 anos & Uganda \\
\hline Buriti et. al., 2013 & Transversal & 23 & Até 11 anos & Brasil \\
\hline Gregory et. al., 2013 & ----- & 14 & A partir de 8 anos & Brasil \\
\hline Buriti et. al., 2014 & Transversal & 23 & 2 a 10 anos & Brasil \\
\hline Torre et. al.,2015 & ----- & 61 & A partir de 4 anos & África do Sul \\
\hline Romero et. al.,2017 & ----- & 15 & 8 e 9 anos & Brasil \\
\hline Nakku et. al.,2017 & Transversal & 227 & 6 a 12 anos & Uganda \\
\hline Peter et. al., 2020 & Exploratório descritivo & 30 & 6 a 12 anos & África do Sul \\
\hline
\end{tabular}


Os métodos de avaliação e alterações no sistema auditivo encontrados estão apresentados na Tabela 2. Os resultados da presente revisão indicaram que sete $\operatorname{artigos}(70 \%)$ descreveram perda auditiva do tipo condutivacomo alteração em crianças com AIDS (Taipale et. al., 2011); (Chao et. al., 2012); (Gregory et. al., 2013); (Buriti et. al., 2014); (Torre et. al., 2015); (Nakku et. al., 2017); (Peter et. al. 2020). Já a perda auditiva do tipo neurossensorial foi relatada em três estudos (30\%) (Ndoleriire et. al., 2013); (Nakku et. al., 2017); (Torre et. al., 2015). Dois artigos (20\%) apontaramproblemas a nível de processamento auditivocentral (Gregory et. al., 2013); (Romero et. al.,2017) e três (30\%) estudos encontraram perfuração timpânica (Peter et. al., 2020); (Taipale et.al., 2011); (Chao et. al., 2012).

Para avaliação auditiva, sete $(70 \%)$ dos estudos utilizaram audiometria tonal e impedanciometria(Gregory et. al., 2013); (Buriti et. al., 2013); (Taipale et. al., 2011); (Peter et. al., 2020); (Buriti et. al., 2014); (Nakku et. al., 2017); (Chao et. al.,2012)e três (30\%) utilizaram ainda o PEATE/BERA (Taipale et. al., 2011); (Ndoleriire et. al., 2013); Peter et. al., 2020).

Tabela 2. Descrição dos métodos de avaliação e alterações auditivas encontradas nos estudos selecionados.

\begin{tabular}{|c|c|c|c|}
\hline AUTOR/ANO & OBJETIVO & MÉTODO DE AVALIAÇÃO & $\begin{array}{c}\text { ALTERAÇÕES NO SISTEMA } \\
\text { AUDITIVO }\end{array}$ \\
\hline Taipale et. al., 2011 & $\begin{array}{l}\text { Definir e relacionar as manifestações } \\
\text { otorrinolaringológicas em crianças com } \\
\text { HIV positivo e negativo, em Luanda. }\end{array}$ & $\begin{array}{l}\text { PEATE/BERA } \\
\text { Audiometria tonal ao ar livre }\end{array}$ & $\begin{array}{c}\text { Otite } \\
\text { Perfuração timpânica } \\
\text { Perda auditiva condutiva }\end{array}$ \\
\hline Chao et. al., 2012 & $\begin{array}{c}\text { Determinar a prevalência de PA na } \\
\text { síndrome da imunodeficiência adquirida } \\
\text { em crianças que vivem no Peru. }\end{array}$ & $\begin{array}{l}\text { Audiometria tonal } \\
\text { Imitanciometria }\end{array}$ & $\begin{array}{l}\text { Perda auditiva condutiva } \\
\text { Perfuração timpânica }\end{array}$ \\
\hline $\begin{array}{l}\text { Ndoleriire C. et al., } \\
2012\end{array}$ & $\begin{array}{c}\text { Verificar a incidência, tipos de PA em } \\
\text { crianças com HIV entre } 6 \text { meses e } 5 \\
\text { anos de idade acolhidos no PIDC, } \\
\text { Hospital MulagoUganda. }\end{array}$ & PEATE/ BERA & Perda auditiva neurossensorial \\
\hline Buriti et. al., 2013 & $\begin{array}{c}\text { Determinar a prevalência de PA em } \\
\text { crianças com HIV/AIDS e identificar } \\
\text { sua relação com a } \\
\text { carga viral, as doenças oportunistas e a } \\
\text { técnica antirretroviral estabelecida. }\end{array}$ & $\begin{array}{l}\text { Audiometria tonal condicionada } \\
\text { Imitanciometria }\end{array}$ & $\begin{array}{c}\text { Otite } \\
\text { Perda auditiva súbita e progressiva }\end{array}$ \\
\hline Gregory et. al., 2013 & $\begin{array}{c}\text { Analisar a audição periférica e central } \\
\text { de crianças com HIV, acompanhamento } \\
\text { audiológico e auxílio de orientações e } \\
\text { práticas para os acadêmicos de } \\
\text { fonoaudiologia. }\end{array}$ & $\begin{array}{l}\text { Audiometria tonal } \\
\text { Audiometria vocal } \\
\text { Imitanciometria } \\
\text { PEATE de longa latência }\end{array}$ & $\begin{array}{l}\text { Perda auditiva condutiva } \\
\text { Alterações no processamento } \\
\text { auditivo central }\end{array}$ \\
\hline Buriti et. al., 2014 & $\begin{array}{l}\text { Analisar a audição de crianças com } \\
\text { HIV/AIDS, relacionando à idadee ao } \\
\text { período de diagnóstico. }\end{array}$ & $\begin{array}{l}\text { Audiometria tonal } \\
\text { Imitanciometria }\end{array}$ & Perda auditiva condutiva \\
\hline Torre et. al., 2015 & $\begin{array}{l}\text { Buscar dados audiológicos em crianças } \\
\text { com infecção perinatal por HIV e não } \\
\text { infectadas, mas expostas ao HIV, } \\
\text { residentes em Cabo, África do Sul. }\end{array}$ & $\begin{array}{l}\text { Audiometria tonal } \\
\text { EOAPD }\end{array}$ & $\begin{array}{c}\text { Perda auditiva condutiva e } \\
\text { neurossensorial }\end{array}$ \\
\hline Romero et. al.,2017 & $\begin{array}{c}\text { Descrever o as funções auditivas de } \\
\text { crianças infectadas pelo HIV através do } \\
\text { Teste de Processamento Auditivo } \\
\text { Simplificado e Teste } \\
\text { StaggeredSpondaic Word (SSW). }\end{array}$ & $\begin{array}{l}\text { Teste de Processamento Auditivo } \\
\text { Simplificado } \\
\text { StaggeredSpondaic Word }\end{array}$ & $\begin{array}{c}\text { Alteração de Processamento } \\
\text { auditivo }\end{array}$ \\
\hline Nakku et. al., 2017 & $\begin{array}{c}\text { Comparar a incidência de PA em } \\
\text { crianças positivas e negativas para HIV, } \\
\text { estabelecer os tipos e se a terapia } \\
\text { antirretroviral (ART) está concernente a } \\
\text { ela. }\end{array}$ & Audiometria Tonal & $\begin{array}{c}\text { Perda auditiva condutiva e } \\
\text { neurossensorial }\end{array}$ \\
\hline Peter et. al., 2020 & $\begin{array}{c}\text { Identificar o perfil auditivo de uma rede } \\
\text { de crianças com idade escolar } \\
\text { apresentando HIV/AIDS e em } \\
\text { acompanhamento médico em uma } \\
\text { Clínica de ARV. }\end{array}$ & $\begin{array}{l}\text { Audiometria tonal } \\
\text { Audiometriavocal } \\
\text { Imitanciometria } \\
\text { EOA } \\
\text { PEATE/BERA }\end{array}$ & $\begin{array}{l}\text { Perda auditiva do tipo condutiva } \\
\text { Perfuração Timpânica }\end{array}$ \\
\hline
\end{tabular}

Legenda: PEATE/BERA - Potencial evocado auditivo de tronco cerebral; EOA - Emissões Otoacústicas; EOAPD - Emissões otoacústicas por produto de distorção.Fonte: Autores. 
Devido a importância da integridade do sistema auditivo periférico e central para o desenvolvimento de fala e linguagem, assim como socialização e desenvolvimento da aprendizagem torna-se fundamental a realização detestes audiológicos para monitoramento e detecção precoce de anormalidades auditivasna população pediátrica.

Nesta revisão sistemática, os resultados dos estudos analisados foram consistentes, indicando alterações no sistema auditivo periférico e central, sendo utilizada por eles como principal método de avaliação do sistema periféricoa audiometria tonal, audiometria vocal e imitanciometria. Os resultados destes exames, em sua maior parte, apontaram perda auditiva do tipo condutiva como alteração. Essa condição é explicada pelo fato destes indivíduos estarem sujeitos a infecções como as de ouvido médio (Taipale et. al., 2011); (Peter et. al., 2020); (Buriti et. al., 2014); (Romero et. al., 2014); (Nakku et.al., 2017); Gregory et.al., 2013). Em relação aos tipos de otite encontrados três estudos detectaram otite média (Buriti et. al., 2013); (Peter et.al., 2020); (Romero et.al., 2017) e dois otite média suprativa crônica (Taipale et.al., 2011); ( Ndoleriire et. al., 2013).

Embora os achados na literatura apresentem-se com maior incidência em perdas auditivas do tipo condutiva, um estudo realizado na Clínica Pediátrica de Doenças Infecciosas, no Hospital Mulago em Uganda, que teve como população amostral 374 pacientes, com idade entre 6 meses e 5 anos constatou a prevalência de perda auditiva do tipo sensorioneural (64\%) causando perdas piores em direção as altas freqüências (Ndolerire et. al. 2013). É importante ressaltar que as crianças com AIDS fazem uso de medicação contínua, podendo esta ser ou não ototóxica, como o TARV (Chao et. al., 2012). Desta forma, o uso de medicações ototóxicas pode justificar a perda auditiva do tipo sensorioneural.

Em estudo comparativo sobre a perda auditiva em indivíduos que apresentam HIV e que não apresentam HIV, autores apontam maior incidência de perda auditiva no grupo com HIV em relação ao grupo que não apresentam a doença, isso pode se justificar devido à predisposição que essas pessoas têm de desenvolver otites, e também pela exposição a medicamentos que aumentam essa predisposição (Chao et al., 2012); (Torre et al., 2015). No entanto, outra pesquisa traz dados divergentes (Nakku et. al., 2017), onde a ocorrência de perda auditiva se apresenta de forma semelhante entre esses dois públicos, porém não descreve claramente a discrepância desse resultado.

Segundo White-Schwoch et. al. (2020) além de atingir o sistema auditivo periférico, a atividade do vírus pode ocasionar também comprometimento progressivo nas estruturas do sistema nervoso central, mesmo com terapia antirretroviral eficaz. Existem várias hipóteses responsáveis por esse comprometimento, incluindo inflamação crônica, dano persistente da infecção aguda, efeitos neurotóxicos do antirretroviral e estresse oxidativo de uma cascata de ativação do sistema imunológico. De acordo com Zhan et. al. (2018), o sistema nervoso central (SNC) pode servir como um reservatório anatômico para o HIV e áreas relacionadas ao processamento auditivo provavelmente estão envolvidas.

A integridade do sistema auditivo central é imprescindível para o desenvolvimento de tarefas auditivas e aprendizagem. Com o intuito de examinar este sistema, Gregory et. al. (2013) utilizou como método de avaliação o teste Potenciais Evocados Auditivos de Tronco Encefálico (PEATE) de média e/ou longa latência. Neste estudo, 14 crianças e adolescentes com AIDS foram submetidas ao PEATE, exame que analisa atividade neuroelétrica da via auditiva central. Constatou-se que todas as crianças apresentaram alterações nas respostas da avaliação do potencial evocado auditivo de longa latência e do potencial cognitivo nos quesitos de atenção, discriminação e memória auditiva.

Seguindo o mesmo objetivo e utilizando testes diferentes, o estudo de Romero et. al. (2017) buscou analisar o processamento auditivo central de 15 crianças com idade entre 8 e 9 anos em uso de medicamento antirretrovirais. A avaliação baseou-se em dois testes comportamentais: o Teste de Processamento Auditivo Simplificado (SAPT) e o Staggered Spondaic Word (SSW). No primeiro teste $60 \%$ das crianças apresentaram dificuldades em processar as informações. Já na segunda avaliação mais da metade dos participantes apresentaram indicativas de déficit nas habilidades auditivas. 
Diante do levantamento bibliográfico, observou-se a escassez de estudos que relatem achados audiológicos e tipos de perda auditiva em crianças portadoras do vírus da imunodeficiência. Verifica-se que crianças e adolescentes com AIDS são passíveis, em diversos períodos de diagnóstico a complicações auditivas de diversos tipos.

\section{Conclusão}

As principais alterações auditivas a nível periférico em crianças e adolescentes portadores da Síndrome da Imunodeficiência Adquirida (AIDS) descritas na literatura são: perda auditiva do tipo condutiva, otite e perfuração timpânica.Comprometimentos a nível de Processamento Auditivo Central também foram descrito em alguns estudos.

Diante do exposto é notória a necessidade de realizar exames audiológicos para acompanhamento da saúde auditiva de crianças e adolescentes com AIDS. A detecção precoce de alterações da audição seja a nível central ou periférico é um fator relevante para o pleno desenvolvimento comunicacional nessa população.

\section{Referências}

Buriti, A. K. L., Oliveira, S. H. S.,\&Muniz, L. F. M. (2013). Hearingloss in childrenwith HIV/AIDS. Revista Codas [online]. doi.org/10.1590/S2317-17822013.05000013

Buriti, A. K. L., Oliveira, S. H. S, Muniz, L. F. M.,\&Soares, M. J. G. O. (2014). Evaluationofhearing in childrenwith HIV/ AIDS. Audiology Communication Research. doi.org/10.1590/S2317-64312014000200002

Chao, C. K., Czechowicz, J. A., Messner, A. H., Alarcón, J., Kolevic Roca, L., Larragán Rodriguez, M. M., Gutiérrez Villafuerte, C., Montano, S. M., \&Zunt, J. R. (2012). High prevalence of hearing impairment in HIV-infected Peruvian children. Otolaryngology--head and neck surgery: official journal of American Academy of Otolaryngology-Head and Neck Surgery, 146(2), 259-265. doi.org/10.1177/0194599811429271

Costa, A. R., Nobre, C. M. G., Gomes, G. C., Ribeiro, J. P., Mota, M. S., \&Minasi, A. S. A. (2020). Mudanças no viver a partir do diagnóstico da criança/adolescente com HIV/AIDS e facilidades encontradas no cuidado. Research, Society and Development, 9(7), e55973707. doi.org/10.33448/rsd-v9i7.3707

De-la-torre-ugarte-guanilo, M. C.; Takahashi, R. F.; \&Bertolozzi, M. R.(2011). Revisão sistemática: noções gerais. Revista da Escola de Enfermagemda USP, . 45(5): 1260-1266.doi.org/10.1590/S0080-62342011000500033

Gregory, L., Cardoso, E., Bonfada, L., \&Sleifer, P. (2013). Avaliação Audiológica e monitoramento em crianças infectadas com HIV. Int. Arco. Otorhinolaryngol. (Suplemento 1);81.

Hortúa B. A. F., Montero C. J. ,Oñate C. S., C. V. C.,\&Cuadrado C. B. (2020). Caracterización de lãs manifestaciones otorrinolaringológicas en pacientes coninfección por VIH/sida enlaciudad de Cartagena. Acta otorrinolaringol. cir. cabezacuello; 48(1): 283-290. 10.37076/acorl.v48i4.548

Matas, C. G., Samelli A. G., Magliaro F. C., \&Segurado A. (2017). Audiological and electrophysiological alterations in HIV-infected individual s subjected or not to antiretroviral therapy. Brazilian Journal of Otorhinolaryngology84:574-82.doi.org/10.1016/j.bjorl.2017.07.003

Maro, II, Fellows, A. M., Clavier, OH, Gui, J., Rieke, C. C., Wilbur, J. C., Chambers, R. D.,Jastrzembski, B. G.,Mascari, J. E., Bakari, M.,Matee, M.,Musiek, F. E., Waddell, R. D., von Reyn, C. F., Palumbo, P. E., Moshi, N.,\&Buckey, J. C. (2016). Deficiências auditivas em crianças infectadas pelo HIV. Ouvido e audição 37 (4), 443-451. doi.org/10.1097/AUD.0000000000000276

Minhas, R. S., Dhanashree A. IyengarJagdeep S. \&ThakurRamesh K. Azad(2018). Effect of HIV and Antiretroviral Treatment on Auditory Functions. International Archives of Otorhinolaryngologydoi.org/10.1055/s-0038-1639594

Nakku, D., Nyaiteera, V., Llowet, E., Nanseera, D., Nakalema, G., Westerberg, B., \&Bajunirwe, F. (2017). HIV status and hearing loss among children between 6 and 12 years of age at a large urban health facility in south western Uganda. International journal of pediatric otorhinolaryngology, 101, 172177. doi.org/10.1016/j.ijporl.2017.08.011

Ndoleriire, C., Turitwenka, E. ,Bakeera K. S.,\&Nyabigambo A.(2013) The prevalence of hearing impairment in the 6 months -5 years HIV/AIDS-positive patients attending paediatric infectious disease clinic at Mulago Hospital. International Journal of Pediatric Otorhinolaryngology. doi.org/10.1016/j.ijporl.2012.11.013

Oluwabusayo D. B., Adebolajo A.A., \&Regina E. O. (2018). Otolaryngologic Lesions among Human Immunodeficiency Virus-infected Children. Ann OtolNeurotolISO 1:105-110

Peter, V., Paken, J.,\&Joseph, L. (2020). An audiological profile of a cohort of school-aged children with HIV and AIDS attending an antiretroviral clinic in South Africa.South African Journal of Communication Disorders, 67 (1), p.9.

doi.org/10.4102/sajcd.v67i1.651

Quidicomo, S. e M., \& Carla G. (2013). Estudo da função auditiva em indivíduos com HIV/AIDS submetidos e não submetidos à terapia antirretroviral. Audiology - Communication Research: 18(1), 10-16. 
Research, Society and Development, v. 11, n. 1, e9811124730, 2022

(CC BY 4.0) | ISSN 2525-3409 | DOI: http://dx.doi.org/10.33448/rsd-v11i1.24730

Romero, A. C.,Alfaya, L. M, Gonçales, A. S.,Frizzo, A. C.,\&Isaac, M. L. (2017). Auditory alterations in children infected by human immunodeficiency virus verified through auditory processing test. Int ArchOtorhinolaryngol, 21 (1), 86-91. doi.org/10.1055/s-0036-1580614

Santos, N. de A., Arcanjo Matos Filho, S., Santos Matos, D., \&Lordelo Carvalho, T. R. (2019). Desenvolvimento de um aplicativo android para prevenção da síndrome da imunodeficiência adquirida (AIDS). Revista Saúde.Com, 15(2). doi.org/10.22481/rsc.v15i2.4731

Sebothoma, B., \&Shangase K. K. , (2018). A comparison between video otoscopy and standard tympanometry findings in adults living with human immunodeficiency virus (HIV) in South Africa. South African Journal of Communication Disorders, 65 (1),1 7. doi.org/10.4102/sajcd.v65i1.591

Smith, A. F., Ianacone, D. C., Ensink, R., Melaku, A., Casselbrant, M. L., \&Isaacson, G. (2017). Prevalence of hearing-loss among HAART-treated children in the Horn of Africa. International journal of pediatric otorhinolaryngology, 98, 166-170. doi.org/10.1016/j.ijporl.2017.04.050

Taipale, A., Pelkonen, T., Taipale, M., Roine I., Bernardino, L., Peltola, H., \&Pitkaranta, A. (2011). Otorhinolaryngological findings and hearing in HIVpositive and HIV-negative children in a developing country. Eur Arch Otorhinolaryngol 268, 1527.doi.org/10.1007/s00405-011-1579-x

Torre, P. 3rd, Cook, A., Elliott, H., Dawood, G., \& Laughton, B. (2015). Hearing assessment data in HIV-infected and uninfected children of Cape Town, South Africa. AIDS care, 27(8), 1037-1041. doi.org/10.1080/09540121.2015.1021746

White-Schwoch T.,Magohe A. K., Fellows A. M., Rieke C. C., Vilarello B., Nicol T.,Massawe E. R, Moshi N, Kraus N, \&Buckey J. C. (2020) Auditory neurophysiology reveals central nervous system dysfunction in HIV-infected individuals. ClinNeurophysiol. Aug;131(8):1827-1832. 10.1016/j.clinph.2020.04.165

Zhan, Y., Fellows, A. M., Qi, T., Clavier, O. H., Soli, S. D., Shi, X., Gui, J., Shi, Y., \& Buckey, J. C. (2018). Speech in Noise Perception as a Marker of Cognitive Impairment in HIV Infection. Ear and hearing, 39(3), 548-554. doi.org/10.1097/AUD.0000000000000508 